Case Report

\title{
Plasmapheresis for the Treatment of Iatrogenic Antithrombin Excess as a Result of Spuriously Low Antithrombin Activity Measurements
}

\author{
Reza Zarinshenas, Eric D. Destrampe, and William Nicholas Rose \\ Department of Pathology and Laboratory Medicine, University of Wisconsin Hospital and Clinics, 600 Highland Ave, Madison, \\ WI 53792, USA
}

Correspondence should be addressed to William Nicholas Rose; wrose@uwhealth.org

Received 8 February 2020; Accepted 1 May 2020; Published 8 May 2020

Academic Editor: Mehmet Doganay

Copyright () 2020 Reza Zarinshenas et al. This is an open access article distributed under the Creative Commons Attribution License, which permits unrestricted use, distribution, and reproduction in any medium, provided the original work is properly cited.

\begin{abstract}
We report an intensive care patient who was given excessive amounts of antithrombin concentrate in response to spuriously low antithrombin activity measurements and was then treated with plasmapheresis (therapeutic plasma exchange or TPE) to remove the excess.
\end{abstract}

\section{Introduction}

Antithrombin (AT) is a key physiological inhibitor of coagulation in vivo [1]. AT prevents the expansion and formation of clots [2]. AT inactivates thrombin, plasmin, and other serine proteases [1]. The effect of AT is increased approximately 1000-fold in the presence of heparin [3]. Recombinant or human AT is approved for the treatment and prevention of thromboembolism in those with hereditary AT deficiency $[4,5]$. Human AT has an in vivo halflife of approximately 2.5-3.8 days, a molecular weight of about $58,000 \mathrm{kDa}$, and a high bioavailability in the plasma $[4,6]$. There appears to be no literature on the acute removal of extremely high levels of AT.

\section{Case Presentation}

2.1. Patient Data. A 63-year-old female was admitted with acute liver failure of unknown etiology complicated by stage III acute kidney injury due to presumed hepatorenal syndrome. Her clinical status was complicated by toxic/metabolic encephalopathy, coagulopathy, and massive gastrointestinal bleeding with nadir hemoglobin of $4.4 \mathrm{~g} / \mathrm{dL}$. She received an orthotopic liver transplant.
The patient received heparin on three different days. According to our best reading of the intraoperative records, she received 3,000-13,000 units during the transplant. She also received 10,000 units on postoperative day 2 and 5,000 units of heparin on postoperative day 3 . The patient received $2.2 \mathrm{~mL}$ alteplase on postoperative day 2 . The patient also received aspirin in the postoperative period. AT levels were ordered starting postoperative day 0 right after surgery because there was hepatic artery mismatch between the patient and the donor, which raised a concern for an increased risk of hepatic artery thrombosis $[7,8]$.

The AT activity was initially $26-29 \%$. She received antithrombin factor replacement (using GRIFOLS THROMBATE III) on postoperative day 1. After factor replacement, AT levels were reported as being below the lower limit of detection for the assay $(<20 \%)$. She then received additional doses of AT factor replacement. In total, she received approximately 30,000 total units of AT factor replacement from postoperative day 1 to 2 . It was discovered that the reported AT activity levels post replacement were spuriously low (for more details, see Details of Antithrombin Test Results).

The corrected AT values were higher than the upper limit of detection for the assay ( $>150 \%)$. During the AT replacement period, the patient was intubated and exhibited altered 
mental status even during periods when sedation was discontinued. As a consequence, our assessment of her symptoms was limited. For example, we could not assess if she experienced dizziness, chest pain, bowel fullness, chills, cramps, dysgeusia, dyspnea, or nausea. She could open her eyes at times but did not follow commands. There was some oozing from her abdominal incision in the early postoperative period, some passage of dark red blood per rectum postoperatively, and oozing at postpuncture sites. There was no report of hematuria, hemarthrosis, hives, or film over the eye.

The transfusion medicine service was consulted on postoperative day 3 to see if TPE could be performed to remove excess AT, given its half-life of 2.5-3.8 days and concern of a possible increased bleeding risk in some patient populations with high AT levels $[9,10]$. At that time, the patient was thrombocytopenic, had elevated blood urea nitrogen, and had received 5,000 units of heparin earlier that day.

Removal of AT via hemodialysis was not possible due to AT's large size. Since AT has high bioavailability in plasma, TPE was a rational method for its acute removal. To replenish other coagulation factors, plasma was used as replacement fluid in a 1-volume exchange via automated centrifugal plasmapheresis. Briefly, plasmapheresis involves the transporting of a small volume of whole blood from the patient into a machine, the separation of the whole blood via density into plasma and cells, and the simultaneous removal of plasma and infusion of a replacement fluid.

The postexchange AT level was $125 \%$ and within the normal range of $83-128 \%$. Subsequent levels were slightly elevated then tapered down into the normal range. A few days after plasmapheresis, she developed bloody stools secondary to a rectal ulcer bleed. She ultimately went to the operating room for oversewing of her bleeding rectal ulcer. Other complications that occurred after the liver transplant included continued altered mental status thought to be caused by metabolic encephalopathy, critical illness neuromyopathy, and respiratory failure necessitating a tracheostomy. Her altered mental status improved during the course of the hospitalization, and she underwent tracheostomy decannulation once her respiratory status stabilized. The patient's overall clinical status improved, and she was discharged to a rehabilitation facility.

2.2. Details of Antithrombin Test Results. All antithrombin levels were measured using the liquid antithrombin automated chromogenic assay by Hemosil. Initially low, but correct, AT levels (26-29\%) prompted administration of AT factor replacement. After the administration of AT, six samples were reported as "failed" by the ACL TOP 700 CTS analyzer (Instrumentation Laboratory). These measurements were taken from postoperative day 1 through postoperative day 3. Laboratory personnel, unaware that the patient was receiving AT factor replacement, mistakenly interpreted these as representing levels below the limit of detection. But in fact, the levels were above the analyzer's detection. Once the error was discovered, the samples were diluted with Hemosil Factor Diluent (1:5 dilution) and rerun for three of the six spuriously low measurements. The three corrected values were determined to be $170 \%, 400 \%$, and $485 \%$. The other three spuriously low values could not be rerun because of sample stability issues (these samples were not frozen). Antigen levels were not checked by the laboratory.

\section{Discussion}

3.1. Antithrombin Laboratory Error. The chromogenic assay to measure AT activity depends on a linear relationship between the reporter signal and time (personal communication with Instrumentation Laboratory). The assay is linear only from 10 to $150 \%$ [11]. For values outside this range, the instrument reports a "failure." Dilution of samples as discussed above is required to give accurate measurements when the failure is due to an activity that is above the upper limit of detection.

3.2. Plasmapheresis Treatment. Since there was no known precedent for using plasmapheresis to remove AT, there was some uncertainty about whether the procedure was indicated. There was no clear evidence that high antithrombin levels increase bleeding risk in the absence of heparin therapy or that there is any other reason to treat high levels of AT. The decision to treat in this case was based on the following considerations. First, the patient had some concerning risk factors for bleeding including thrombocytopenia, an elevated blood urea nitrogen, a recent massive gastrointestinal bleed a few days prior, some bleeding in the postoperative period, just had a liver transplant, and received heparin the same day transfusion medicine was contacted about performing plasmapheresis. Second, there are articles that report that high-dose AT treatment may be associated with an increased risk of bleeding in some patient populations $[9,10]$. Thus, TPE was performed, and the AT activity decreased.

\section{Conclusion}

We report the first case to our knowledge of treating AT excess using TPE. TPE may be a valuable tool for the removal of molecules with these characteristics: a long half-life, a high plasma bioavailability, and a size that is too large for removal by dialysis. The three main points of this report are the following: (1) spuriously low AT results, if unrecognized and unresolved by the laboratory, may lead to unnecessary AT factor replacement and a possible bleeding tendency; (2) a specimen that causes a "failed" run on an AT chromogenic assay may be resolved by diluting and running it again; and (3) plasmapheresis should be considered for the treatment of AT excess.

\section{Conflicts of Interest}

The authors declare they have no conflicts of interest.

\section{References}

[1] M. Hepner and V. Karlaftis, "Antithrombin," Methods in Molecular Biology, vol. 992, pp. 355-364, 2013.

[2] February 2020, https://www.thrombate.com/en/home. 
[3] K. Kottke-Marchant and A. Duncan, "Antithrombin deficiency: issues in laboratory diagnosis," Archives of Pathology \& Laboratory Medicine, vol. 126, no. 11, pp. 1326-1336, 2002.

[4] THROMBATE III ${ }^{\circledR}$ (antithrombin III [human]) [Prescribing Information], Grifols, Therapeutics LLC Research Triangle Park, NC, 2019.

[5] M. M. Patnaik and S. Moll, "Inherited antithrombin deficiency: a review," Haemophilia, vol. 14, no. 6, pp. 1229-1239, 2008.

[6] February 2020, https://reference.medscape.com/drug/ thrombate-iii-antithrombin-iii-342868.

[7] A. A. Algarni, M. M. Mourad, and S. R. Bramhall, "Anticoagulation and antiplatelets as prophylaxis for hepatic artery thrombosis after liver transplantation," World Journal of Hepatology, vol. 7, no. 9, pp. 1238-1243, 2015.

[8] J. Quintero, J. Ortega, M. Miserachs, J. Bueno, I. Bilbao, and R. Charco, "Low plasma levels of antithrombin III in the early post-operative period following pediatric liver transplantation: should they be replaced? A single-center pilot study," Pediatric Transplantation, vol. 18, no. 2, pp. 185-189, 2014.

[9] B. L. Warren, A. Eid, P. Singer et al., "Caring for the critically ill patient. High-dose antithrombin III in severe sepsis: a randomized controlled trial," Journal of the American Medical Association, vol. 286, no. 15, pp. 1869-1878, 2001.

[10] C. J. Wiedermann, J. N. Hoffmann, M. Juers et al., "High-dose antithrombin III in the treatment of severe sepsis in patients with a high risk of death: efficacy and safety," Critical Care Medicine, vol. 34, no. 2, pp. 285-292, 2006.

[11] Liquid antithrombin [package insert], Hemosil, Bedford, Massachusetts, 2017. 